\title{
Grasp Synthesis for Digital Hands with Limited Range of Motion in Their Thumb Joints
}

\author{
Reiko Takahashi, ${ }^{1}$ Natsuki Miyata, ${ }^{2}$ Yusuke Maeda, ${ }^{3}$ and Koji Fujita ${ }^{4}$
}

\begin{abstract}
For virtual evaluation of universal design products, it is necessary to synthesize natural grasps for various hands including those with limited range of motion (ROM). In this paper, we study synthesizing grasps for digital hands with limited ROM in their thumbs' joints. We apply a contactregion-based method for grasp synthesis to this problem. In our previous study, two types of grasps were observed under the limitation of the thumb's ROM. One was a type that used the lateral region of the thumb, and the other was a type that used

the same contact regions as healthy hands. In this paper, grasps are synthesized for two objects using three hand models whose lengths are different. With the same input information as the healthy hand, it is not possible to synthesize the grasps for the ROM-limited hand. On the other hand, when grasps are synthesized by changing the information about grasping target points of the thumb and the palm, feasible grasps for the ROMlimited hand can be found. For a camera object, the grasp using the thumb's radial region is automatically synthesized without changing the specified contact region. Also, for a spray object, a grasp that uses the same contact region as the healthy hand is synthesized. These results demonstrate our method can synthesize natural grasps even for ROM-limited hands, which will contribute to universal product design.
\end{abstract}

\section{INTRODUCTION}

Recently, the evaluation of products virtually using digital human models for human-centered design has been proposed in [1]-[4]. For example, in [1], virtual evaluation of products by estimating grasps for their CAD models using a digital hand was proposed. In the virtual evaluation, usability can be assessed roughly without subject experiments. There are two major merits in such virtual evaluation. One is cost saving. As the number of subject experiments is decreased, the labor cost and the cost of creating prototypes can be reduced. The other is the comprehensive consideration of various hands. If the subjects are actually recruited, their diversity and universality will not be guaranteed. In the virtual evaluation, this problem can be solved by using various digital hands.

For the virtual evaluation using a digital hand, it is necessary to synthesize natural grasps for any product model. Methodologies of grasp synthesis were roughly divided into analytical approaches and empirical ones. By the analytical approaches, the grasp synthesis problem is formulated geometrically, kinematically, and/or mechanically, while by the empirical approaches, the computation of the mathematical

\footnotetext{
${ }^{1}$ Graduate School of Engineering Science, Yokohama National University, Kanagawa, JAPAN, takahashi-reiko-cf@ynu.jp

${ }^{2}$ Digital Human Research Team, National Institute of Advanced Industrial Science and Technology, Tokyo, JAPAN

${ }^{3}$ Div. of Systems Research, Faculty of Engineering, Yokohama National University, Kanagawa, JAPAN

${ }^{4}$ Tokyo Medical and Dental University, Tokyo, JAPAN
}

and physical models is avoided by imitating human grasping strategies. In the analytical approaches, there is a problem on task modeling [5]; that is, adapting grasp synthesis algorithms to new tasks or new objects is difficult because many candidate grasps would have to be generated and evaluated to synthesize appropriate grasps, which may make these approaches computationally unaffordable. In [6], studies about data-driven (empirical) grasp synthesis were surveyed. Examples of data-driven grasp synthesis approaches are [7] [11]. Data-driven approaches use the database of real human grasps generally and the necessary data are identified based on known grasp classification (e.g., [12]-[16]). Therefore it is possible to synthesize natural grasps that are more similar to human real grasps than analytical approaches.

Previous studies on grasp synthesis have been focusing mainly on healthy hands. However, non-healthy hands should also be considered as market size of universal design products expands. Grasps by non-healthy hands differ from those of healthy hands. Thus, in the virtual evaluation of universal design products, it is important to synthesize natural grasps by various hands including non-healthy ones.

Grasps by hands with limited range of motion (ROM) are typical examples of grasps different from those of healthy hands. In particular, because the thumb is used for $98-99 \%$ of the grasps [17], the change of grasp posture becomes noticeable when the ROM of the thumb is limited. Patients with carpal tunnel syndrome (CTS) are a typical example of those whose thumbs' ROM is limited. CTS is the most common peripheral nerve entrapment syndrome worldwide [18]. It is caused by compression of the median nerve at the wrist. Particularly in patients with severe CTS, it is known that the palmar abduction of the carpometacarpal $(\mathrm{CM})$ joint is restricted (e.g., [19]). Since the opposition of the thumb is hindered, significant change in grasping posture of the hands is expected when their palmar abduction of the $\mathrm{CM}$ joint is limited.

We have investigated the grasp features when the ROM of palmar abduction of the thumb's CM joint was artificially limited [21]. In this paper, we present grasp synthesis when the thumb's palmar abduction is limited based on the features clarified in [21]. Realization of grasp synthesis for ROM-limited hands contributes to evaluation of usability of universal design products virtually.

\section{Algorithm OF GRASP SYNTHESIS}

In this paper, the method of grasp synthesis for healthy hands proposed in [10] and [11] is applied to grasp synthesis 
for ROM-limited hands. This section explains an overview of the algorithm of grasp synthesis.

The greatest feature of this method is the use of grasp database based on the "contact regions." The outline of the method of grasp synthesis proposed in [10] and [11] is shown in Fig. 1. The contact regions are the hand surface areas that touch the object when grasping. Hirono et al. divided the hand surface into 34 regions, and classified grasps that appear in the taxonomy proposed in [12] and [13] based on the contact regions. Furthermore, additional grasp postures with different number of used fingers and with different interfingertip distance were selected, and finally, a grasp database including 801 natural grasps was created. The postures with different number of used fingers were added to the grasp database in order to cover expected possible grasps. In addition, the postures with different inter-fingertip distance were added to synthesize the grasp for a newly given object by interpolating a few postures in the grasp database.

The postures in the grasp database were held in the form of joint angles acquired by using an optical motion capture (MoCap) system with 25 reflective markers placed on the dorsal side of the hand. The advantage of the grasp database based on the contact regions is that the posture in the grasp database whose contact regions are same as the input regions can be used to synthesize the initial posture even if the grasped object is different. Therefore, it is expected to have wide applicability to object variations of size and shape.

The input information of grasp synthesis is the contact regions and the points of grasping targets on the object surface. In the method, postures having the same contact regions as the input data are searched from the database firstly. Then, an initial posture is synthesized by interpolating the reference grasps whose contact regions are same as the input data based on the distance between the target points on the object surface. In this step, the hand model is also aligned with the object by matching the contact points on the hand with the corresponding contact target points on the object.

Because the initial posture is not guaranteed to be a natural and proper grasp, the initial posture is adjusted by quasiNewton method. The following five indicators were used for adjustment: forming contact at given regions, forming a contact in appropriate orientation, keeping postures within joint ROM, joint coordination, and self-interference avoidance. Forming contact at given regions is considered to ensure that the specified contact regions reliably contact the object surface. Forming a contact in appropriate orientation is considered so that the normal vector of the hand is directed in parallel with that of the object when grasping. Keeping postures within joint ROM is considered to ensure that the joints' angles of the posture are feasible. If a joint angle is out of range, returning torque is added to the joint. Joint coordination is considered to ensure that the combination of the joints' angles is also feasible. Instead of considering only the maximum and minimum values of the joint angles, three parameters are defined for each joint and their mutual dependency is considered in this indicator. Finally, self-

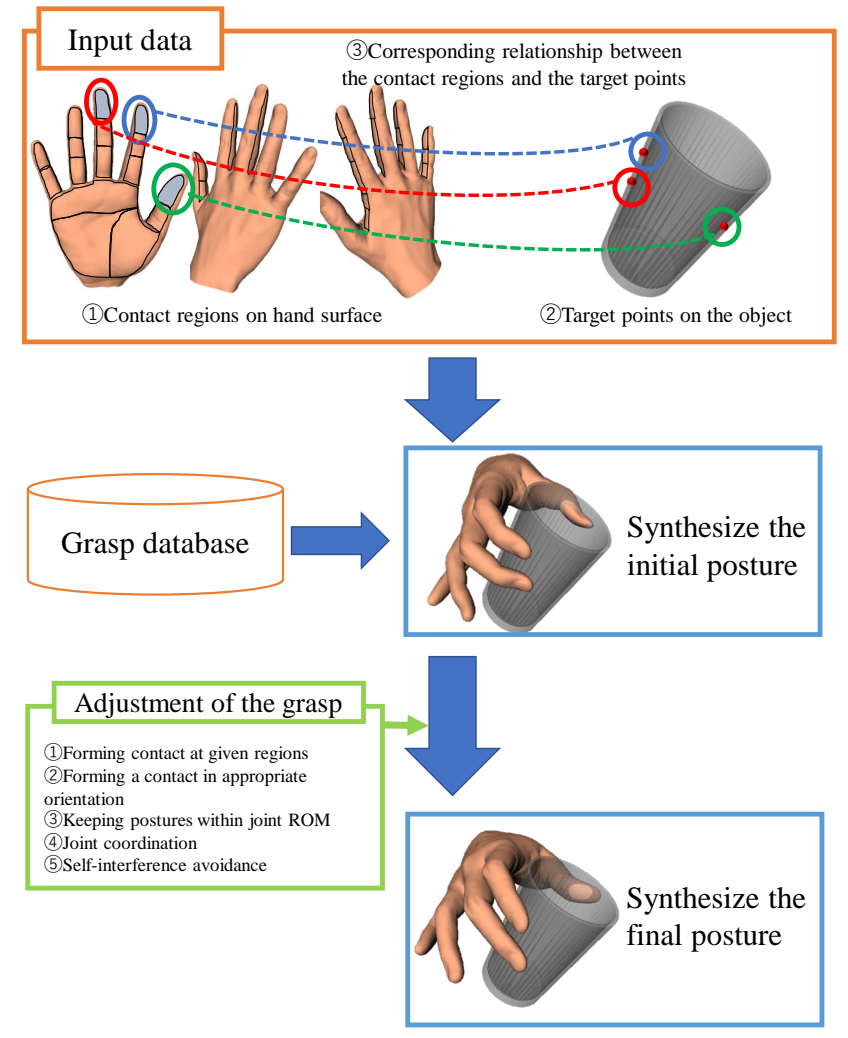

Fig. 1. Outline of the method of grasp synthesis proposed in [10] and [11]

interference avoidance is considered to ensure the avoidance of interference among the fingers or the palm. The hand model is divided into 18 rigid bodies and the interference is checked for all pairs of segments using proximity query package [20].

\section{The FEATURES OF LIMITED ROM}

This paper aims to synthesize the grasps for thumb's ROM-limited hands by adding the information of the ROM limitation to the database of healthy hands proposed by [10] and [11]. As another approach, it is possible to create a new database for ROM-limited hands from scratch. However, it is necessary to acquire a huge amount of postures and rebuild the database for each type of ROM-limitation. In anticipation of increased types of limitation dealt with in future work, grasps are synthesized by adding minimal changes to the database of healthy hands.

In [21], we measured the ROM when the palmar abduction of thumb's CM joint was artificially limited using our proposed taping method to emulate CTS, and investigated the grasp characteristics of an artificially ROM-limited hand. There was no limitation in the radial direction of the thumb's CM joint, but the radial abduction was limited due to the limitation of palmar abduction, because there is a strong mutual dependency between the palmar abduction and the radial abduction of the thumb's CM joint. Since individual differences among ROMs are very large even in healthy 


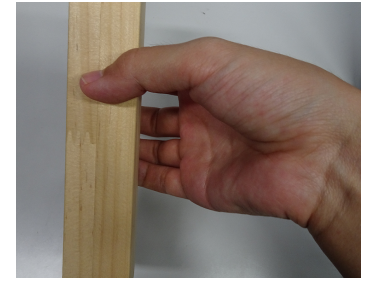

(a) a grasp using thumb's ulnar side

Fig. 2. Examples of grasps using thumb's lateral region (often found in grasps by ROM-limited hands)

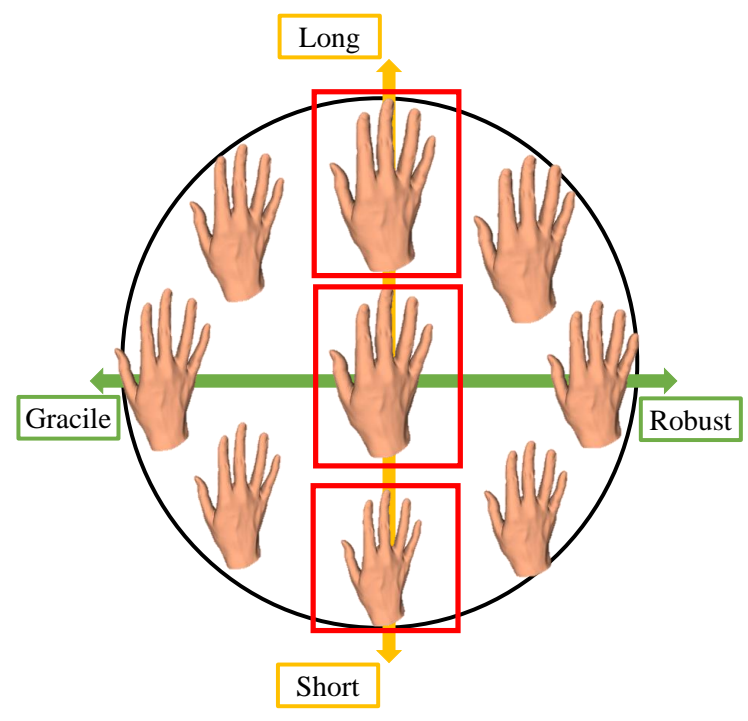

Fig. 3. Representative nine hand models proposed in [22]

hands, only the rough features of the measured limited ROM in [21] were considered in the ROM limitation for the grasp synthesis in this paper. In the coordinate system of the hand model used by [10] and [11], it was confirmed that the ROM limitation by the taping method proposed in [21] restricted about $60 \%$ of the palmar abduction and $40 \%$ of the radial abduction.

In the method of grasp synthesis proposed in [10] and [11], the ROM information is used only when adjusting the posture in optimization calculation (the indicators of keeping postures within joint ROM and joint coordination). In this study, as a method with the least change from [10] and [11], ROM-limited hand's grasps are synthesized simply by narrowing the ROM (60\% decreased palmar abduction and $40 \%$ decreased radial abduction of the CM joint). The quality of synthesized grasps is verified.

In the experiments in our study that follows [21], it was found that there were two types of grasps with the ROMlimited hands. One was a type that used the lateral region of the thumb (Fig. 2) as revealed in [21], and the other was a type that used the same contact regions as healthy hands. Whether these features are observed in the synthesized grasp postures are one of the points of relevance of the grasp synthesis method.

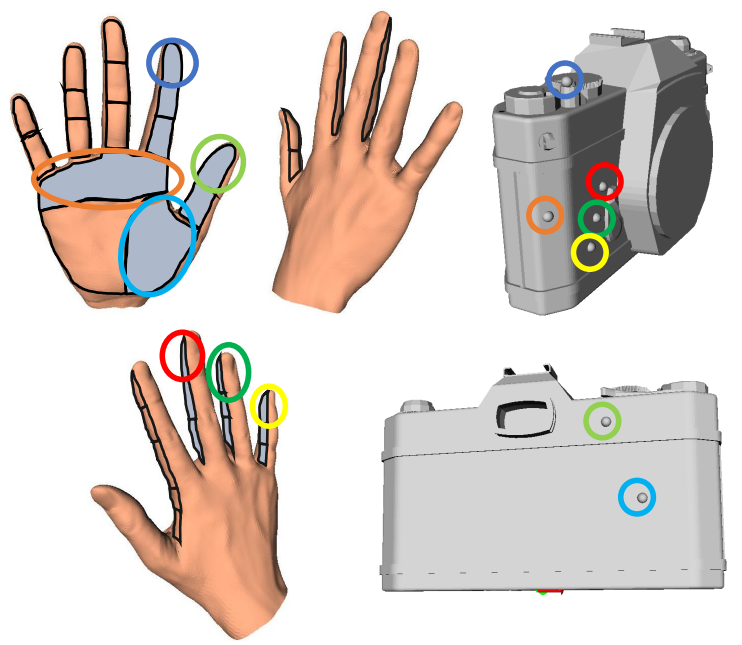

(a) Input data for the model of the camera

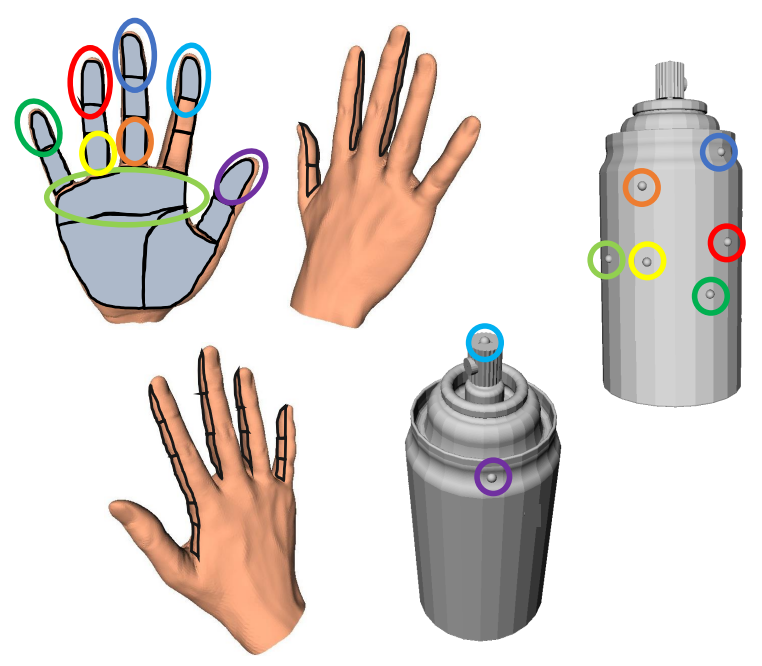

(b) Input data for the model of the spray can

Fig. 4. Input data for grasp synthesis for the three object models

\section{GRASP SYNTHESIS FOR ROM-LIMITED HAND}

\section{A. Using the same input data as the healthy hand}

1) Conditions of the grasp synthesis: The features of the human hands are very diverse. In [22], the thickness of joints and the finger lengths were focused as the characteristics of hands, and the nine representative forms of human hands were shown in terms of two parameters (long/short and robust/gracile) (Fig. 3). In this study, we focused on the finger lengths and used three hand models with different lengths (the hands surrounded by the red line in Fig. 3) for grasp synthesis. Hirono et al. synthesized the grasps by healthy hands whose lengths were different and confirmed that the flexion angle of the fingers varied due to the difference of the lengths [11].

We considered both a healthy hand (with original ROM) and a ROM-limited hand (with narrowed ROM) for each of the three hand models with different lengths. Grasps were 
synthesized for two object models (camera and spray can), and synthesized postures were compared. The contact regions and target points of each object model are shown in Fig. 4. The gray regions on the hand surface indicate the contact regions, and circles with the same color indicate a target point on the object and its corresponding contact region.

2) Results and Discussions: The postures synthesized for the camera by the hand model whose length is long are shown in Fig. 5, and those for the spray can by the hand model whose length is short are shown in Fig. 6 as the examples of results. In either case, no significant difference in the postures can be confirmed between the healthy and ROM-limited hands. In the posture for the camera, however, the thumb obviously interferes with the grasped object for the ROM-limited hand. (Note the area surrounded by the green circle in Fig. 5 (b).) If the tip of the thumb attempts to approach the target point within the narrowed ROM, it is considered that the thumb cannot move to the position along the object surface. It is assumed that the position of the thumb's target point is inadequate. On the other hand, for the spray can, the unnatural posture in which the index finger is not in contact with the grasped object is synthesized for the ROM-limited hand. (Note the area surrounded by the green circle in Fig. 6 (b).) While the angle between the thumb and the index finger is adjusted by the thumb's CM joint for the healthy hand, that for the ROM-limited hand is adjusted by the angle of the index finger because the thumb's ROM is narrowed. Therefore, the posture of the index finger tends to be unfeasible. These results indicate that synthesis of feasible grasps for ROM-limited hands by inputting the same contact regions and grasping target points as the healthy hand is difficult.

\section{B. Changing the input data for the ROM-limited hand}

1) Conditions of the grasp synthesis: In the real observation of the grasps by ROM-limited hand, the big difference between the posture for the ROM-limited hand and that for the healthy hand is the contact position of the thumb and palm with the object surface. Therefore, grasp synthesis is attempted by excluding the information of the thumb's grasping target points and changing the palm's target points from the input data. In the grasp synthesis method proposed by Miyata et al., it is not necessary to specify grasping target points for all selected contact regions. Taking advantage of this feature, grasp synthesis is attempted under the condition that the thumb is allowed to contact the object at any position.

The change of the input data for the ROM-limited hand is shown in Fig. 7. The same regions as the healthy hand are specified as the contact regions, and only the grasping target points are changed. The color of the circle indicating the original target point corresponds to Fig. 4. The changes are as follows:

- Exclusion of the thumb's grasping target points

- Moving the grasping targets of the palm regions to the proximal and ulnar direction

- Adding the target point corresponding to the proximal palm region (if the target point is not given to the
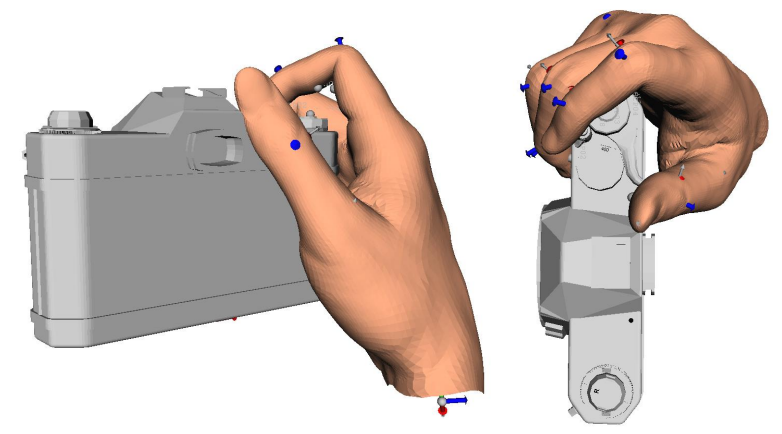

(a) Grasp posture synthesized for the healthy hand
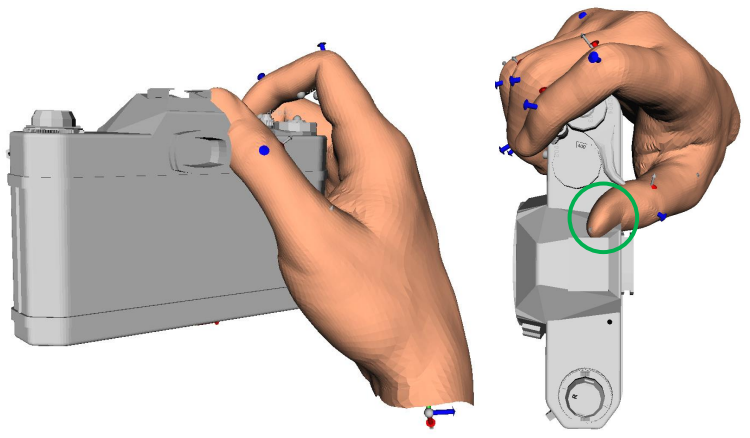

(b) Grasp posture synthesized for the ROM-limited hand

Fig. 5. Grasp postures for the camera: hand model whose length is long

proximal palm region in the healthy hand)

2) Results and discussions: The posture synthesized for the camera by the ROM-limited hand model whose length is long is shown in the Fig. 8, and that for the spray can by the ROM-limited hand model whose length is short is shown in Fig. 9. By changing the input data, interference with the object of the thumb and separation of the index finger from the object are avoided, and the feasible postures are synthesized for the ROM-limited hand. Comparing Fig. 8 to Fig. 5 (a), it can be obviously seen that the difference between the thumb's positions as surrounded by the blue circle in Fig. 8. The thumb does not separate from the index finger and the thumb's radial region contacts with the object surface in the posture synthesized for the ROM-limited hand. This feature is consistent with the utilization of the side of the thumb that observed in actual grasps by ROM-limited hands, and the feasible grasp of ROM-limited hand can be synthesized. Next, comparing Fig. 9 to Fig. 6 (a), the differences is smaller as compared with the posture for the camera. However, as shown by the blue circle in Fig. 9, the middle, ring and little fingers wrap around the object shallowly, and the visible area of the palm is wider in the ROM-limited hand. In addition, looking at the posture from the top of the object, back of the hand looks like getting thinner in the posture for the ROM-limited hand because the wrist angle changes. These are the changes that occur 

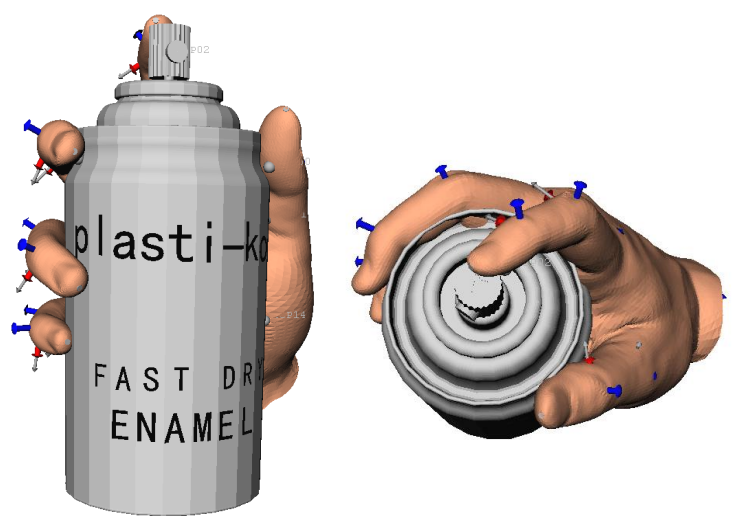

(a) Grasp posture synthesized for the healthy hand
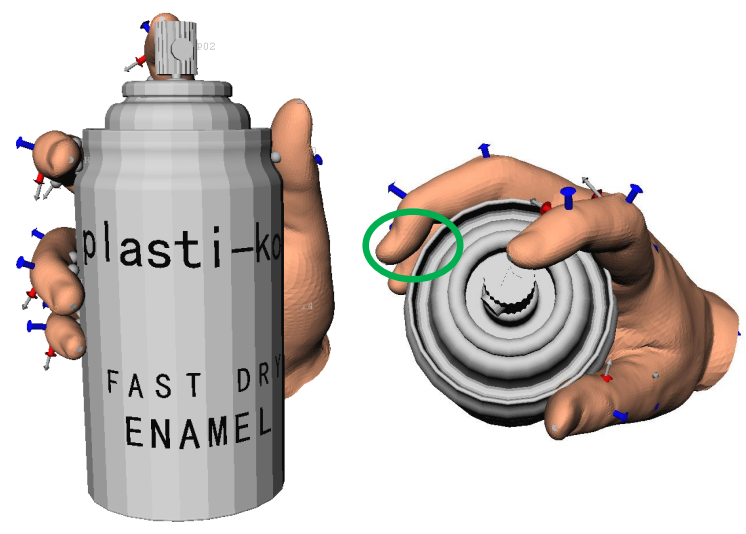

(b) Grasp posture synthesized for the ROM-limited hand

Fig. 6. Grasp postures for the spray can: hand model whose length is short

because the entire hand is closer to perpendicular to the object than the posture for the healthy hand in that for the ROM-limited hand. The contact surface of the thumb is not different from that of the healthy hand. Such grasps are observed also in actual grasps by ROM-limited hands.

The feasible grasp postures for the ROM-limited hand are synthesized by not specifying the contact target points of the thumb. For the ROM-limited hand, the solution space for its grasping posture is narrow due to the limitation of the thumb's ROM, and specifying the target points of the thumb makes grasp synthesis difficult. However, if neither the thumb nor proximal palm region has the corresponded target point, grasp synthesis can also be difficult due to the lack of given information. Moreover, since there is a tendency to support the object with the palm regions instead of the thumb in the ROM-limited hand in the observation of the real grasps, it is considered that the feasible grasps are synthesized by designating the target points of the palm regions on the proximal position than the healthy hand.

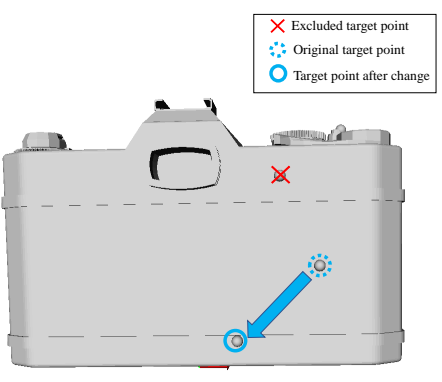

(a) Camera

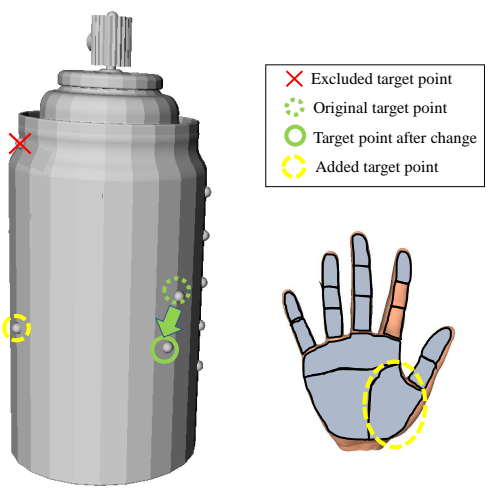

(b) Spray can

Fig. 7. Changing the input data

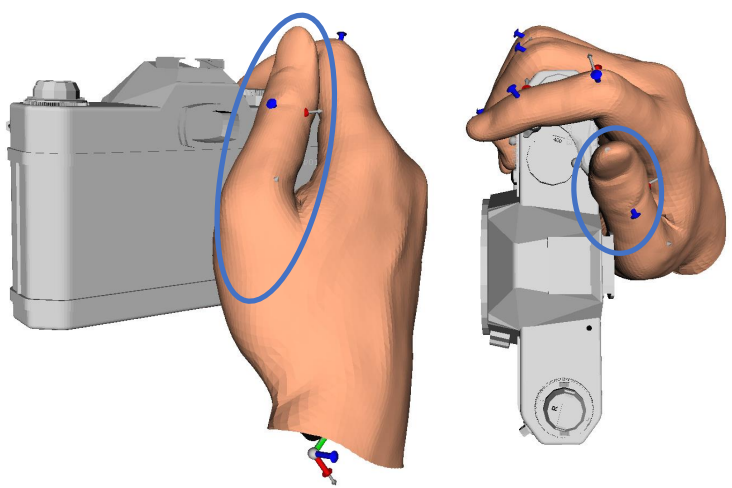

Fig. 8. Grasp postures for the camera excluding the thumb's target point: ROM-limited hand model whose length is long

\section{CONCLUSION}

We tried to synthesize natural grasps for digital hands with limited ROM in their thumbs' joints. Our previous method [10] and [11], originally developed for healthy hands, was applied to this problem. In the coordinate system of the hand model used by [10] and [11], it was confirmed that the ROM limitation by the taping method proposed in [21] restricted about $60 \%$ of the palmar abduction and $40 \%$ of the radial abduction. We added the ROM limitation to the hand model used in [10] and [11] and synthesized the grasps. Two objects (camera and spray can) were prepared and three hand models whose lengths are different were used. In the grasping postures synthesized for the ROM-limited hand, interference 


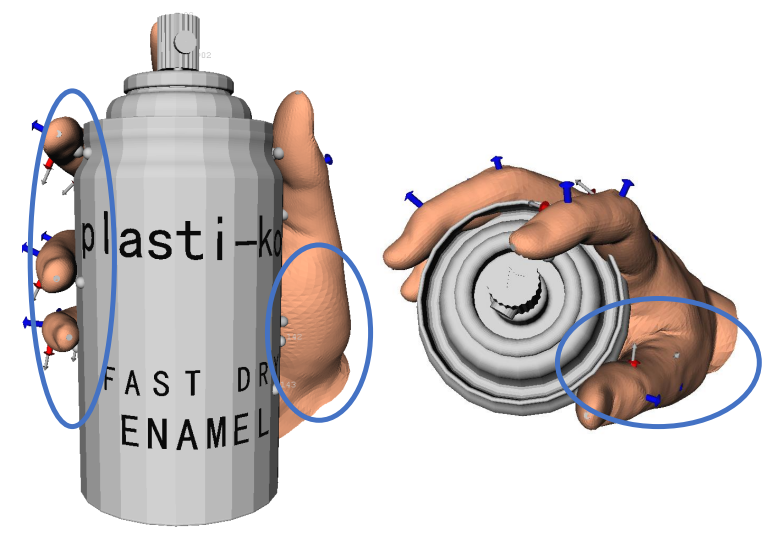

Fig. 9. Grasp postures for the spray can excluding the thumb's target point: ROM-limited hand model whose length is short

of the object with the thumb and noncontact with the object of the index finger occurred.

Then, by excluding the grasping target points of the thumb, moving the grasping targets of the palm regions to the proximal and ulnar direction, and adding the target point corresponding to the proximal palm region, the feasible grasps for the ROM-limited hand were synthesized successfully. In the experiments in our study that followed [21], it was found that there were two types of grasps with the ROM-limited hands. One is the type of grasps whose joint angles of the wrist and the four fingers other than the thumb are adjusted and the contact regions are same as these of the healthy hand. This feature can be reproduced in the synthesized posture for the spray can. The other is the type of grasps in which thumb's lateral region is used. This feature can be reproduced in the synthesized posture for the camera. These results indicate that natural grasps for ROM-limited hands can be synthesized based on grasp database of healthy subjects.

In future work, it is necessary to synthesize a grasp using the thumb's lateral region or a grasp using the same contact regions as that of the healthy hand selectively. Currently either of them is synthesized in our method. However, in the observation of the actual grasps, different types are observed for the same object by each subject. Therefore, synthesizing both types of grasp postures for the same object would be necessary for emulating actual human grasping.

\section{ACKNOWLEDGMENT}

This work was partly supported by JSPS KAKENHI Grant Number JP17H05918.

\section{REFERENCES}

[1] Y. Endo, S. Kanai, T. Kishinami, N. Miyata, M. Kouchi, and M. Mochimaru, An application of a digital hand to ergonomic assessment of handheld information appliances, Proceedings of the SAE Digital Human Modeling for Design and Engineering Conference, 2006, 2006-01-2325.
[2] X. Wang, E. Chateauroux, and N. Chevalot, A data-based modeling approach of reach capacity and discomfort for digital human models, International Conference on Digital Human Modeling, 2007, pp. 215223.

[3] D. Regazzoni, C. Rizzi, and G. Colombo, The role of virtual ergonomic simulation to develop innovative human centered products, Digital Human Modeling. Applications in Health, Safety, Ergonomics and Risk Management: Ergonomics and Health, Vol. 9185, 2015 pp. 74-83.

[4] M. Peruzzini, S. Carassai, M. Pellicciari, and A. O. Andrisano, Human-centred design of ergonomic workstations on interactive digital mock-ups, Advances on Mechanics, Design Engineering and Manufacturing, 2016, pp. 1187-1195.

[5] A. Sahbani, S. El-Khoury, and P. Bidaud, An overview of 3D object grasp synthesis algorithms, Robotics and Autonomous Systems, Vol. 60, No. 3, 2012, pp. 326-336.

[6] J. Bohg, A. Morales, T. Asfour, and D. Kragic, Data-driven grasp synthesis-a survey, IEEE Transactions on Robotics, Vol. 30, No. 2, 2014, pp. 289-309.

[7] F. Kyota, T. Watabe, S. Saito, and M. Nakajima, Detection and evaluation of grasping positions for autonomous agents, International Conference on Cyberworlds, 2005, pp. 453-460.

[8] M. T. Ciocarlie, and P. K. Allen, Hand posture subspaces for dexterous robotic grasping, The International Journal of Robotics Research, Vol. 28, No. 7, 2009, pp. 851-867.

[9] C. Goldfeder, and P. K. Allen, Data-driven grasping, Autonomous Robots, Vol. 31, No. 1, 2011, pp. 1-20.

[10] N. Miyata, T. Hirono, and Y. Maeda, Grasp Database That Covers Variation of Contact Region Usage, 2013 IEEE International Conference on Systems, Man, and Cybernetics, 2013, pp. 2635-2640.

[11] T. Hirono, N. Miyata, and Y. Maeda, Grasp synthesis for variouslysized hands using a grasp database that covers variation of contact region, Proceedings of the 3rd International Digital Human Modeling Symposium, 2014, 11.

[12] T. Feix, R. Pawlik, H.-B. Schmiedmayer, and J. Romero, D. Kragic, A comprehensive grasp taxonomy, Robotics, Science and Systems Conference: Workshop on Understand the Human Hand for Advanced Robotic Manipulation, 2009.

[13] T. Feix, J. Romero, H.-B. Schmiedmayer, A. M. Dollar, and D. Kragic, The GRASP taxonomy of human grasp types, IEEE Transactions on Human-Machine Systems, Vol. 46, No. 1, 2016, pp. 66-77.

[14] J. R. Napier, The prehensile movements of the human hand," The Journal of Bone and Joint Surgery, Vol. 38, No. 4, 1956, pp. 902 913.

[15] N. Kamakura, M. Ohmura, H. Ishii, F. Mitsuboshi, and Y. Miura, Positional patterns for prehension in normal hands, Journal of Rehabilitation Medicine (in Japanese), Vol. 15, No. 2, 1978, pp. 65-82.

[16] K. -S. Lee, and M.-C. Jung, Common patterns of voluntary grasp types according to object shape, size, and direction, International Journal of Industrial Ergonomics, Vol. 44, 2014, pp. 761-768.

[17] F. Gonzalez, F. Gosselin, and W. Bachta, Analysis of hand contact areas and interaction capabilities during manipulation and exploration, IEEE Transactions on Haptics, Vol. 7, No. 4, 2014, pp. 415-429.

[18] L. Padua, D. Coraci, C. Erra, C. Pazzaglia, I. Paolasso, C. Loreti, P. Caliandro, and L. D. Hadson-Webb, Carpal tunnel syndrome: clinical features, diagnosis, and management, The Lancet Neurology, Vol. 15, No. 12, 2016, pp. 1273-1284.

[19] T. L. Marquardt, R. Nataraj, P. J. Evans, W. H. Seitz Jr, and Z.-M. Li, Carpal tunnel syndrome impairs thumb opposition and circumduction motion, Clinical Orthopaedics and Related Research, Vol. 472, No. 8 , 2014, pp. 2526-2533.

[20] Geometric Algorithms for Modeling, Motion, and Animation: “A Proxmity Query Package," http://gamma.cs.unc.edu/SSV/.

[21] R. Takahashi, N. Miyata, Y. Maeda, and K. Fujita, Grasps under Artificially-limited Thumb's Joint Range of Motion -Posture Analysis with ROM Boundary and Muscle Loads-, Proceedings of 2018 International Symposium on Micro-NanoMechatronics and Human Science, 2018, pp. 253-255.

[22] M. Kouchi, N. Miyata, and M. Mochimaru, An analysis of hand measurements for obtaining representative Japanese hand models, Proceedings of the SAE Digital Human Modeling for Design and Engineering Symposium, 2005, 2005-01-2734. 